\title{
Safely mimicking cold exposure to reverse obesity
}

Obesity is caused by an imbalance between energy intake and energy expenditure. Although most current obesity therapies aim to reduce calorific intake, increasing cellular energy expenditure represents an attractive alternative approach. However, attempts to pharmacologically increase energy expenditure have been hindered by the counter-regulatory induction of orexigenic feeding circuits and harmful side effects such as excessive heat production. Writing in Nature Communications, Tschöp and colleagues now report that combinatorial targeting of thermogenic and anorexic pathways safely and synergistically reverses diet-induced obesity and associated complications in mice.

Cold exposure is well-known to increase energy expenditure through the promotion of brown adipose tissue (BAT) thermogenesis. Tschöp and colleagues therefore first focused on the transient receptor potential (TRP) channel, TRPM8, which is a regulator of the cold-sensing cascade that leads to the induction of BAT thermogenesis to defend body temperature in response to environmental cold. In diet-induced obese (DIO) mice, subcutaneous administration of the small-molecule TRPM8 agonist icilin for 14 days lowered body weight and fat mass through an increase in energy expenditure, effects that were not seen in TRPM8-deficient mice.

Given the well-described effect of smoking to suppress hunger, the authors next hypothesized that targeting the nicotinic acetylcholine receptor (nAChR) subtype $\alpha 3 \beta 4$ might counteract any compensatory induction of feeding following TRPM8 agonism. In addition, nAChR activation also acts centrally to lower body temperature.

Subcutaneous administration of the $\mathrm{nAChR}$ a3 34 agonist dimethylphenylpiperazinium (DMPP) alone for 14 days dose-dependently lowered body weight in DIO mice by suppressing food intake and markedly

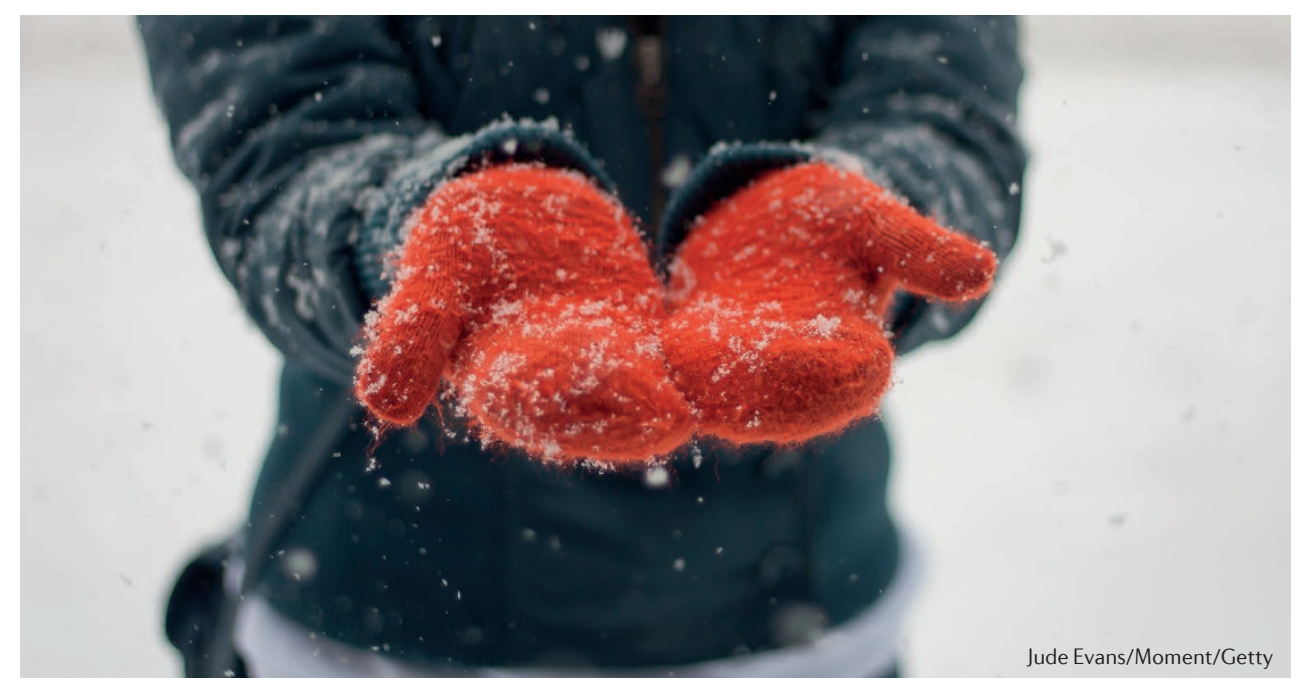

improved diet-induced glucose intolerance. These effects were not seen in nAChR a $3 \beta 4$-deficient mice.

Importantly, acute or chronic treatment of DIO mice with the combination of icilin and DMPP as a single formulation synergistically lowered body weight, an effect that coincided with increased energy expenditure and a robust reduction in food intake.

In addition, the combination therapy corrected diet-induced glucose intolerance in the mice following 1 week of treatment and enhanced insulin sensitivity. Furthermore, while both DMPP and icilin monotherapies improved diet-induced nonalcoholic fatty liver disease and nonalcoholic steatohepatitis, the effect of the cotreatment was superior. Notably, the beneficial metabolic effects of DMPP and icilin were independent of environmental temperature.

Mechanistically, the combination of icilin and DMPP increased neuronal activity in the paraventricular nucleus in mice. Further investigation involving melanocortin 4 receptordeficient mice demonstrated that the melanocortin pathway is indispensable for the weight-lowering and glucose-lowering benefits of the cotreatment. Additional studies using knockout mouse models indicated that thermogenic pathways utilizing sympathetic nervous system-driven activation of $\beta$-adrenergic receptors and uncoupling protein 1 mediate the effects of DMPP and icilin on energy expenditure and weight loss.

In summary, this study indicates that simultaneous targeting of TRPM8 and nAChR $\alpha 3 \beta 4$ may potentially represent a safe approach to pharmacologically mimic the beneficial metabolic effects of cold exposure for the treatment of obesity and associated disorders.

\section{Sarah Crunkhorn}

ORIGINAL ARTICLE Clemmensen, C. et al. Coordinated targeting of cold and nicotinic receptors synergistically improves obesity and type 2 diabetes. Nat. Commun. 9, 4304 (2018) 